\title{
Towards a Telescope Array 10 Year FD Monocular Energy Spectrum
}

\author{
Greg Furlich* and Douglas R. Bergman for the Telescope Array Collaboration ${ }^{\dagger}$ \\ High Energy Astrophysics Institute and Department of Physics and Astronomy, University of \\ Utah, Salt Lake City, UT, USA \\ E-mail: greg.furlich@cosmic.utah.edu
}

The Telescope Array (TA) cosmic ray observatory in Millard County, Utah has passed 10 years of Fluorescence Detector (FD) operation with its 3 detector sites. These FD sites image the cascade of particles created by a cosmic ray entering the atmosphere. This cascade of particles excites the Nitrogen in the atmosphere which in turn emits UV light which is collected by the FDs. With the increased statistics of over 10 years of data, TA is working on reducing the systematic uncertainties of cosmic ray analyses. To better understand the cosmic ray events and TA's FD responses, we introduce a new parameter, $R_{\mathrm{X}_{\max }}$, the distance from the FD site to the event's $X_{\max }$ along the shower core. Incorporating these methods of reducing systematic uncertainties, TA is working on a 10 year monocular energy spectrum to compare with the spectra from the Pierre Auger cosmic ray observatory and TA's surface detectors.

36th International Cosmic Ray Conference -ICRC2019-

July 24th - August 1st, 2019

Madison, WI, U.S.A.

\footnotetext{
* Speaker.

${ }^{\dagger}$ for collaboration list see PoS(ICRC2019)1177
} 


\section{Introduction}

Telescope Array (TA) cosmic ray observatory is the largest cosmic ray detector in the northern hemisphere designed to detect ultra high energy cosmic rays (UHECRs) [1]. It's predocessor, HiRes, was the first cosmic ray observatory to confirm the existence of the supression of cosmic rays at the highest level known as the GZK supression[2] and did so using monocular fluorescence detection. TA has been operating for more than 10 years now and work is being done to update it's monocular energy spectrum.

\section{Telescope Array Cosmic Ray Observatory}

TA is located in Millard County, Utah (Figure 1) due to the location's dry desert air and distance from light pollution sources. TA has 3 FD sites composed of a series of mirrors looking 3 to 33 degrees in elevation and 120 degrees azimuth on the sky to collect fluorescence light produced by Nitrogen excited by the particle cascade in the atmosphere caused by the initial cosmic ray. These FDs sense and track the cosmic ray Extensive Air Shower (EAS) with photomultiplier tubes clustered in a 16x16 grid for each set of mirror segments. TA also has 507 surface detectors arranged in a grid with $1.2 \mathrm{~km}$ spacing to detect the foot print of the the cosmic ray EAS.

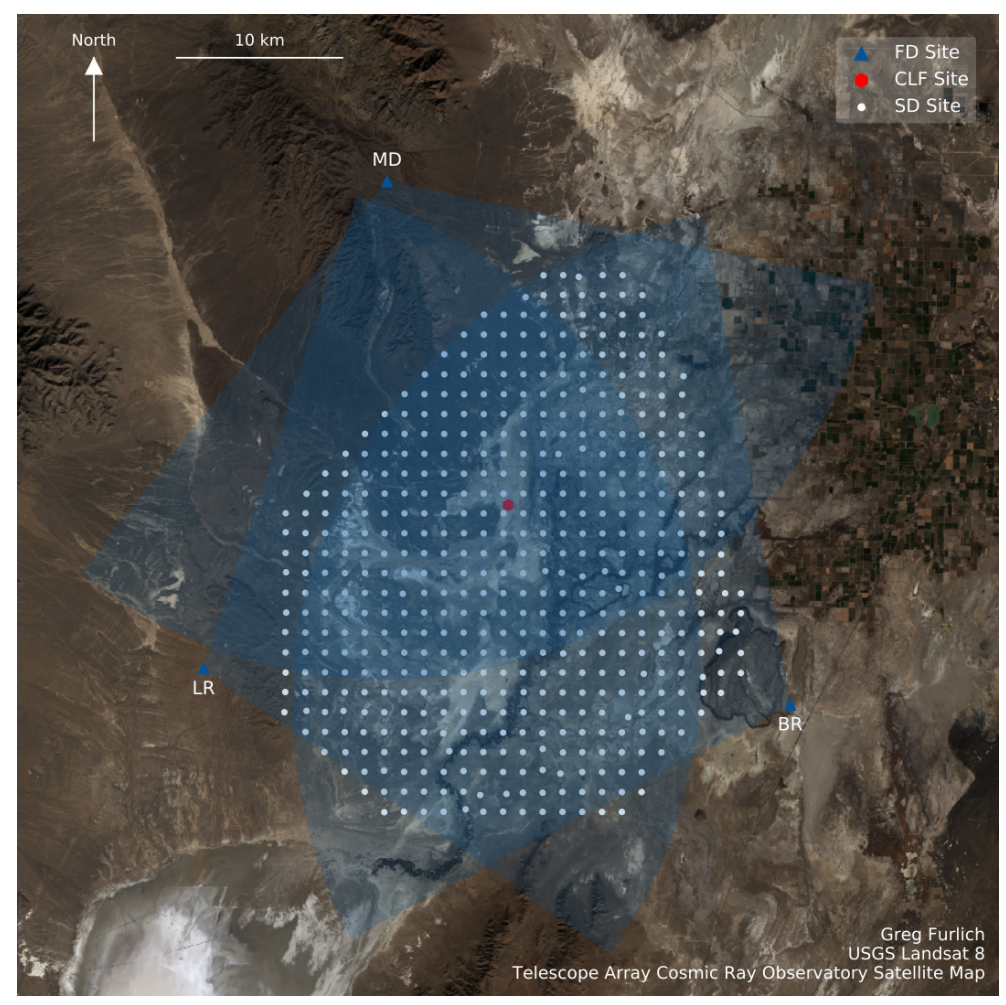

Figure 1: Satellite map of Telescope Array cosmic ray observatory using images from the Landsat 8 satellite. The satellite image was overlayed with the Fluorescence Detector (FD) sites (blue triangle) with a shaded $30 \mathrm{~km}$ field of view (FOV) looking over 507 Surface Detector sites (white circles) and the Central Laser Facility (CLF) (red hexagon). 


\section{Cosmic Ray Event $R_{\mathbf{X}_{\max }}$ Parameter}

To better understand the response of the FDs to an event, we introduce a new parameter, $R_{X_{\max }}$. This parameter is the distance from the FD site to the the shower's maximum development along it's core as shown in Figure 2. Figure 2 also illustrates the difference between $R_{\mathrm{X}_{\max }}$ vs $R_{p}$ which is a construct of the geometry reconstruction and not connected the development of the EAS.

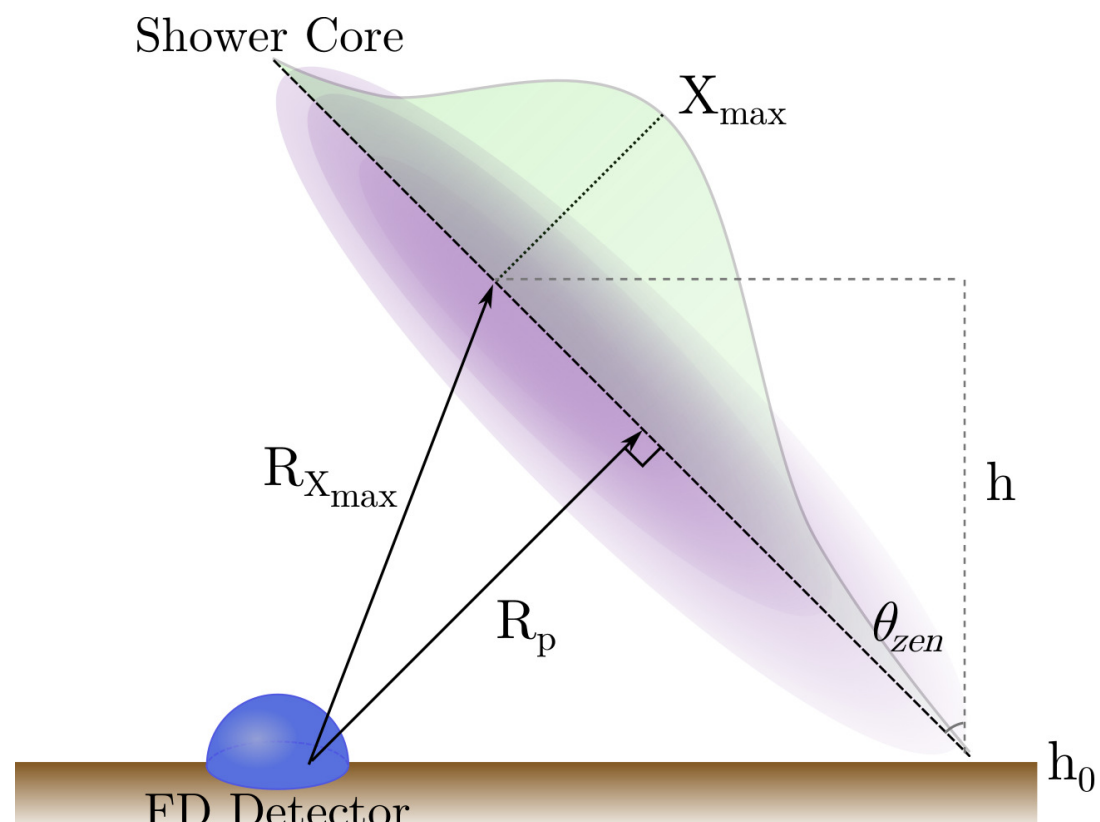

Figure 2: $R_{\mathrm{X}_{\max }}$ parameter vs shower impact parameter, $R_{p}$, for a fluorescence event with the shower development overlaid on top of the shower core.

\subsection{Calculating $R_{\mathbf{X}_{\max }}$}

To calculate $R_{\mathrm{X}_{\max }}$ we used the equation

$$
h\left(X_{\max }, \theta, h_{o}\right)=X^{-1}\left[X_{\text {top }}-X_{\max } \cos \left(\theta_{\text {zen }}\right)\right]-h_{o}
$$

where

$$
X_{\text {top }}=1033.223645 \mathrm{~g} / \mathrm{cm}^{2},
$$

$X^{-1}$ is the inverse function from depth to height calculated numerically using the density of the atmosphere from the US Standard Atmosphere 1976[5], $\theta_{\text {zen }}$ is the zenith angle of the shower core, $h_{o}$ is the FD site's ground level altitude above sea level. Using $h$ and the zenith and azimuth angles of the shower core, the $x$ and $y$ location of $X_{\max }$ with respect to the shower core impact can be determined. Then the distance between the detector site and $X_{\max }$ can be calculated to ultimately determine $R_{\mathrm{X}_{\max }}$. 


\section{10 years of Telescope Array Fluorescence Detection}

\subsection{Operation Time}

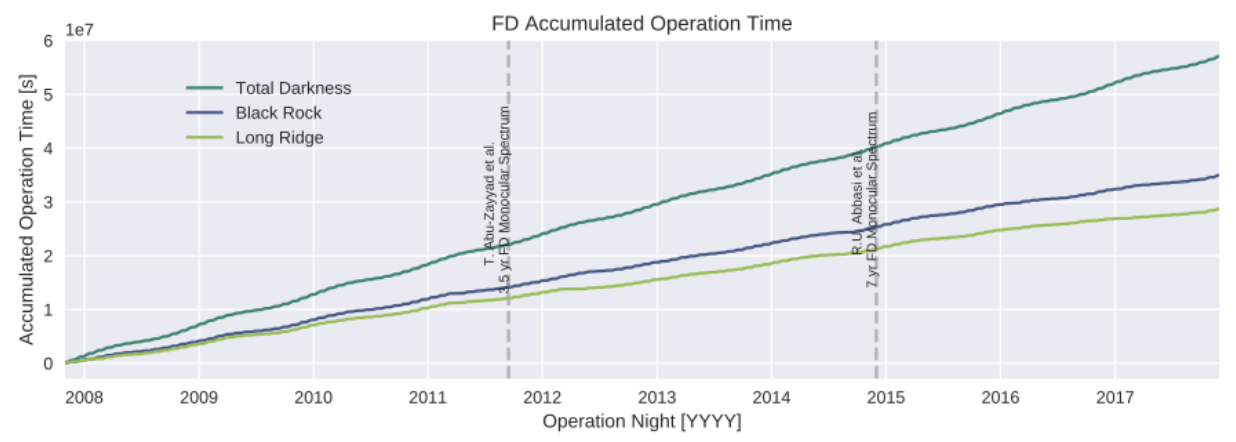

Figure 3: Accumulated operation time of the Black Rock and Long Ridge FD sites over 10 years of operation.

TA gathered first light in late 2007 and began good quality operation in 2008. Figure 3 shows the accumulated operation time. BR and LR operated at a $11 \%$ and $9 \%$ duty cycle respectively over those years. LR operates less than BR due to it's remoteness and the possibility of needing to access it before sunrise should issues arise. The gray lines represent past FD monocular analyses for 3.5 and 7 years which we compare to this work $[3,4]$.

\subsection{Monocular Reconstruction and Quality Cuts}

The first step in monocular reconstruction is determining the plane the shower core makes with the triggered PMTs in the FD. The Shower Detector Plane (SDP) is fit by minimizing the function

$$
\chi^{2}=\sum_{i=1}^{N_{\mathrm{good}}}\left(\hat{n} \cdot \hat{v_{i}}\right)^{2} N_{\mathrm{pe}, i}
$$

to determine the best $\hat{n}$, normal vector of the SDP, with respect to the nominal pointing direction of all the PMTs for the event relative to the to the FD site, $\hat{v}_{i}$ weighted by the amount of photoelectrons, $N_{\mathrm{pe}, i}$, each PMT produces. Once the SDP is determined, the direction of the shower core in the SDP is determined by the timing fit of the good PMTs with the function

$$
t_{i}=t_{0}+\frac{R_{p}}{c}\left[\frac{\pi-\psi-\alpha_{i}}{2}\right]
$$

where $R_{p}$ is the impact parameter, $\psi$ is the inclination angle between the shower core and the detector, and $\alpha_{i}$ is the pointing direction of each PMT in the event. Lastly, the calormetric energy deposited in the atmosphere by the EAS is reconstructed by fitting the shower profile with the Gaisser Hillas function[6],

$$
N_{\mathrm{ch}}(X)=N_{\max }\left(\frac{X-X_{0}}{X_{\max }-X_{0}}\right)^{\frac{X_{\max }-X_{0}}{\lambda}} \exp \left(\frac{X_{\max }-X}{\lambda}\right)
$$


where $N_{\mathrm{ch}}(X)$ is the number of charged particles that develop at slant depth, $X, N_{\max }$ is the maximum number of charged particles produced when the slant depth reaches $X_{\max }$. The Giasser Hillas function is a good phenomenological description of the shower development and the energy deposited in the atmosphere.

To eliminate events that do not reconstruct well, we applied the following quality cuts and kept the events with qualities as listed in the Table 1.

Table 1: FD monocular event reconstruction quality cuts for geometry and profile fitting.

Event Geometry Cuts

\begin{tabular}{|c|c|}
\hline \multicolumn{2}{|l|}{ Successful Timing Fit } \\
\hline Good PMT Fraction & $N_{\text {Good PMT }} / N_{\text {PMTs }} \geqslant 3.5 \%$ \\
\hline Number Good PMTs & $N_{\text {Good PMT }} \geqslant 6$ Tubes \\
\hline NPE per Degree & $N_{\mathrm{pe}} / \Delta \theta>25 \mathrm{NPE} / \mathrm{deg}$. \\
\hline Psuedo Distance & $r_{p}>1.5 \mathrm{~km}$ \\
\hline SDP Angle & $\leqslant 80^{\circ}$ \\
\hline$R_{p}$ & $R_{p} \geqslant .5 \mathrm{~km}$ \\
\hline$\psi$ & $\psi<130^{\circ}$ \\
\hline$\psi$ fit uncertainty & $\sigma_{\psi}<36^{\circ}$ \\
\hline Tangent Fit & $\chi^{2} /$ ndf $<10$ \\
\hline Track Length 1 Ring & $\Delta \theta_{\text {Ring } 1}>7^{\circ}$ \\
\hline Track Length 2 Ring & $\Delta \theta_{\text {Ring } 2}>10^{\circ}$ \\
\hline Zenith Angle & $\theta_{\text {zen }}<70^{\circ}$ \\
\hline Crossing Time & $t_{0}<25.6 \mu \mathrm{s}$ \\
\hline Time Duration & $\Delta t>6 \mu \mathrm{s}\left(\right.$ for $\left.R_{p}<5 \mathrm{~km}\right)$ \\
\hline \multicolumn{2}{|c|}{ Event Profile Reconstruction Cuts } \\
\hline \multicolumn{2}{|l|}{ Successful Profile Fit } \\
\hline First Depth & $150 \mathrm{~g} / \mathrm{cm}^{2} \leqslant X_{1} \leqslant 1200 \mathrm{~g} / \mathrm{cm}^{2}$ \\
\hline Observed Depth Extent & $\Delta X \geqslant 150 \mathrm{~g} / \mathrm{cm}^{2}$ \\
\hline$X_{\max }$ Bracketing & $X_{\max }$ is contained within the FOV \\
\hline
\end{tabular}

\subsection{FD Cosmic Ray Event Distribution}

After the events are reconstucted and the cuts are applied, the remaining events produce Figure 4 event distribution. The distribution agrees qualitatively with the shape of TA's previous monocular analyses $[3,4]$. 


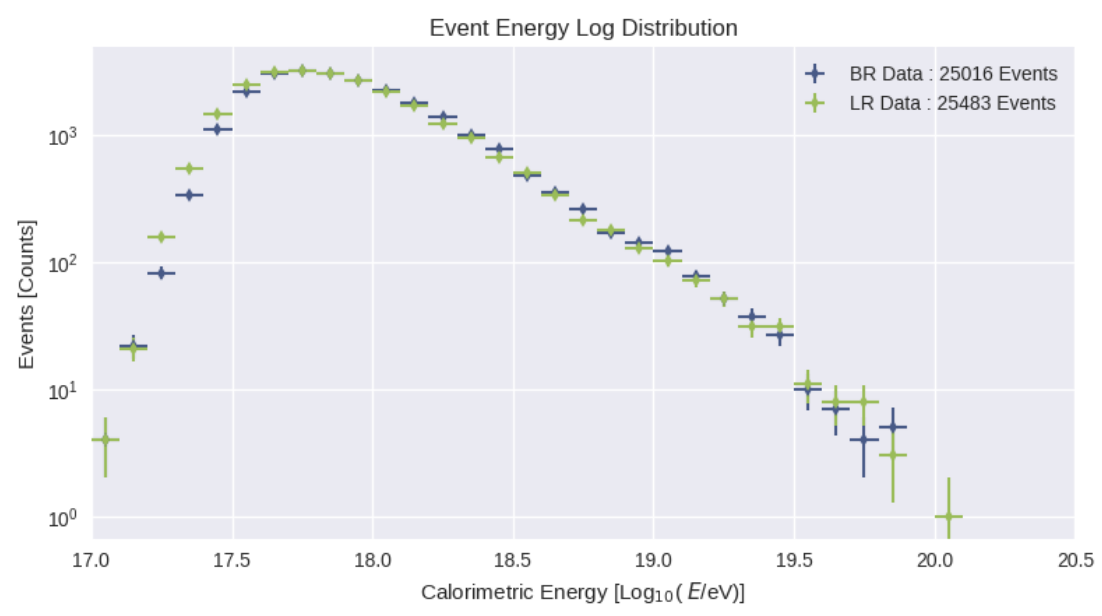

Figure 4: 10 years of FD reconstructed cosmic ray events observed by BR and LR.

\subsection{1 $R_{\mathbf{X}_{\max }}$}

The distribution in Figure 5 of the newly introduced parameter, $R_{X_{\max }}$, also shows agreement between the two FD sites.

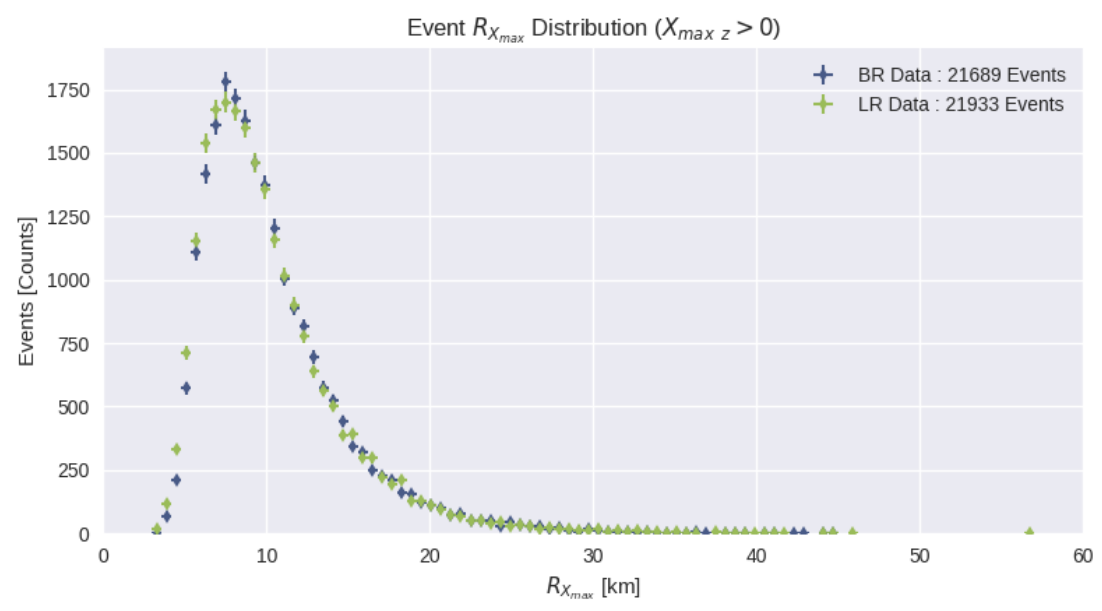

Figure 5: $R_{\mathrm{X}_{\max }}$ Distribution

And looking where the shower core impacts vs the location of $X_{\max }$ calculated while reconstructing $R_{X_{\max }}$, we see the effects of the bracketing cut and better understand the sensitivity of the FDs with this new parameter. 


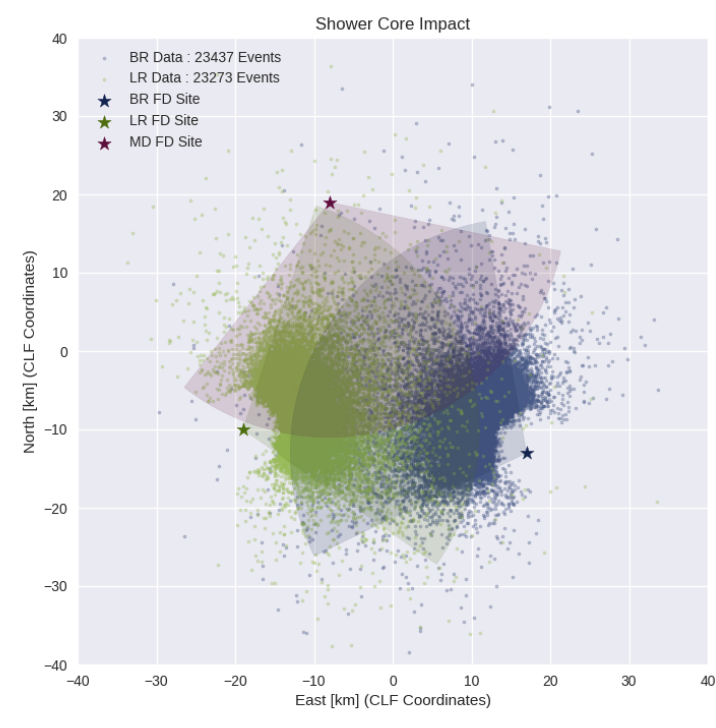

(a) Shower core ground impact

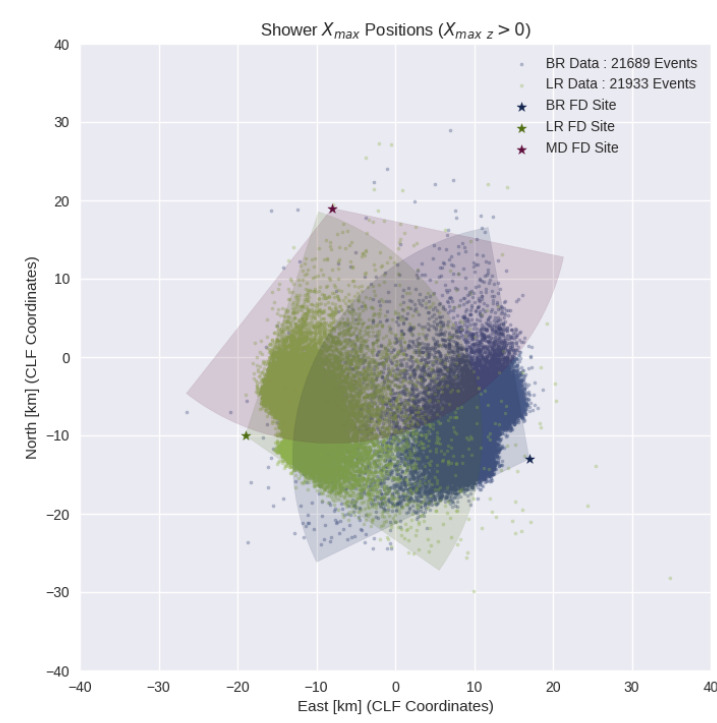

(b) Shower core $X_{\max }$

Figure 6: Event locations out over TA with repect to the FD sites with $30 \mathrm{~km}$ FOV shaded in.

\subsection{Black Rock and Long Ridge Detectors Coincidence Events}

To better understand how well the detectors reconstruct monocular events, coincidence events where both sites reconstructed the same event were found. These coincidence events' characteristics are compared. The energy distributions in Figure $7 \mathrm{a}$ are in good agreement and the energy profile of coincidence in Figure $7 \mathrm{~b}$ shows further that over the energy range these two sites are in agreement of energy reconstruction.

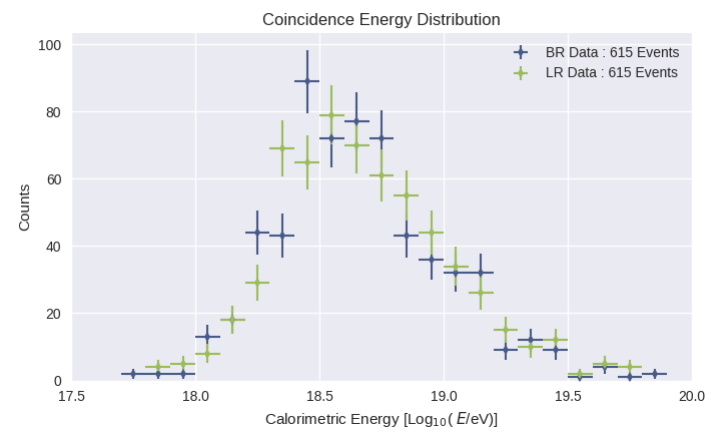

(a) Energy Distribution

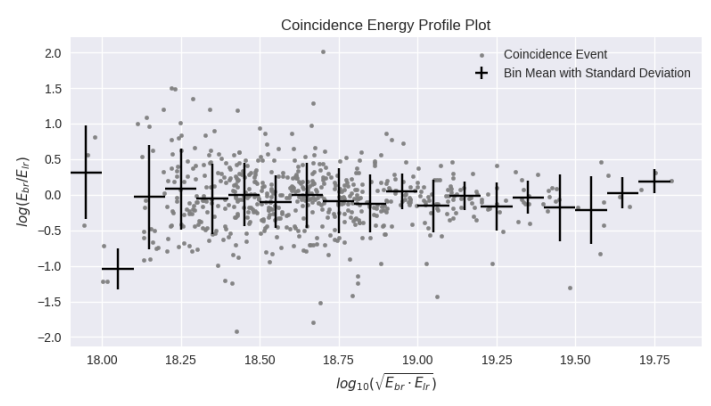

(b) Energy Profile

Figure 7: BR and LR coincidence events

\section{Summary and Plans}

With 10 years of data, an update to TA's FD monocular analysis is underway. Both BR and LR were found to be in agreement with reconstructed events and with the coincidence events seen 
by both sites. A new parameter, $R_{\mathrm{X}_{\max }}$ was introduced to better understand the detectors sensitivity to the shower location. This work will lead to a updated monocular spectrum result from TA which will incorperate the MD detector and apply weather cuts determined using a neural network[7].

\section{References}

[1] H. Kawai et al. for the Telescope Array Collaboration, Nucl.Phys.B, 175-176, 2008, 221-226

[2] R. Abbasi et al. for the Telescope Array Collaboration, Phys.Rev.Lett., 100, 2008

[3] T. Abu-Zayyad et al. for the Telescope Array Collaboration, Astroparticle Phys., 48, 2013, 16-24

[4] R.U. Abbasi et al. for the Telescope Array Collaboration, Astroparticle Phys., 80, 2016, 131-140

[5] U.S. Standard Atmosphere, 1976, U.S. Government Printing Office, Washington, D.C., 1976

[6] T.K. Gaisser, A.M. Hillas, Reliability of the method of constant intensity cuts for reconstructing the average development of vertical showers, in: International Cosmic Ray Conference, 15th ICRC (Plovdiv), vol. 8, 1977, pp. 353-357.

[7] G. Furlich for the Telescope Array Collaboration, these proceedings. 